\title{
Using prediction markets of market scoring rule to forecast infectious diseases: a case study in Taiwan
}

\author{
Chen-yuan Tung ${ }^{1 *}$, Tzu-Chuan Chou ${ }^{2+}$ and Jih-wen Lin ${ }^{3+}$
}

\begin{abstract}
Background: The Taiwan CDC relied on the historical average number of disease cases or rate (AVG) to depict the trend of epidemic diseases in Taiwan. By comparing the historical average data with prediction markets, we show that the latter have a better prediction capability than the former. Given the volatility of the infectious diseases in Taiwan, historical average is unlikely to be an effective prediction mechanism.

Methods: We designed and built the Epidemic Prediction Markets (EPM) system based upon the trading mechanism of market scoring rule. By using this system, we aggregated dispersed information from various medical professionals to predict influenza, enterovirus, and dengue fever in Taiwan.

Results: EPM was more accurate in 701 out of 1,085 prediction events than the traditional baseline of historical average and the winning ratio of EPM versus AVG was $64.6 \%$ for the target week. For the absolute prediction error of five diseases indicators of three infectious diseases, EPM was more accurate for the target week than AVG except for dengue fever confirmed cases. The winning ratios of EPM versus AVG for the confirmed cases of severe complicated influenza case, the rate of enterovirus infection, and the rate of influenza-like illness in the target week were $69.6 \%$, 83.9 and $76.0 \%$, respectively; instead, for the prediction of the confirmed cases of dengue fever and the confirmed cases of severe complicated enterovirus infection, the winning ratios of EPM were all below $50 \%$.
\end{abstract}

Conclusions: Except confirmed cases of dengue fever, EPM provided accurate, continuous and real-time predictions of four indicators of three infectious diseases for the target week in Taiwan and outperformed the historical average data of infectious diseases.

\section{Background}

All public health agencies hope to forecast the outbreak and duration of infectious diseases so that preventive measures can be taken. A popular approach has been the stochastic models, in which the Bayesian models and simulations are some of the advanced methods. These methods are all based on data of infectious diseases collected in a specific time and space, and their reliability is determined by the sample size and their theoretical assumptions. Prediction market, on the other hand, is a mechanism aiming to produce correct forecasts even though the information is partial or limited.

\footnotetext{
* Correspondence: ctung@nccu.edu.tw

${ }^{\dagger}$ Equal contributors

'Graduate Institute of Development Studies, National Chengchi University, 64, Zhi-Nan Road, Sec. 2, Wenshan, Taipei 11605, Taiwan

Full list of author information is available at the end of the article
}

Over the last decade or so, there are 44 studies of epidemic disease prediction models [1]. Except the papers with the prediction markets system written by Polgreen, Nelson, Neumann [2, 3], most forecasting models are based upon the past data and not real-time forecasting models. As Nsoesie, Beckman, Shashaani, Nagarai and Marathe [4] pointed out, those forecasting models could perform well as long as their assumptions are right and there are good surveillance data. Nevertheless, it is difficult to make right assumptions according to different regions around the world and acquire real-time good surveillance data in the real world.

An alternative is to understand the trend of influenza epidemics by search engine query data. Ginsberg et al. [5] and Polgreen et al. [6] used keywords in the search engines of Google and Yahoo to produce trends that are strikingly similar to the flu surveillance pattern. However, 


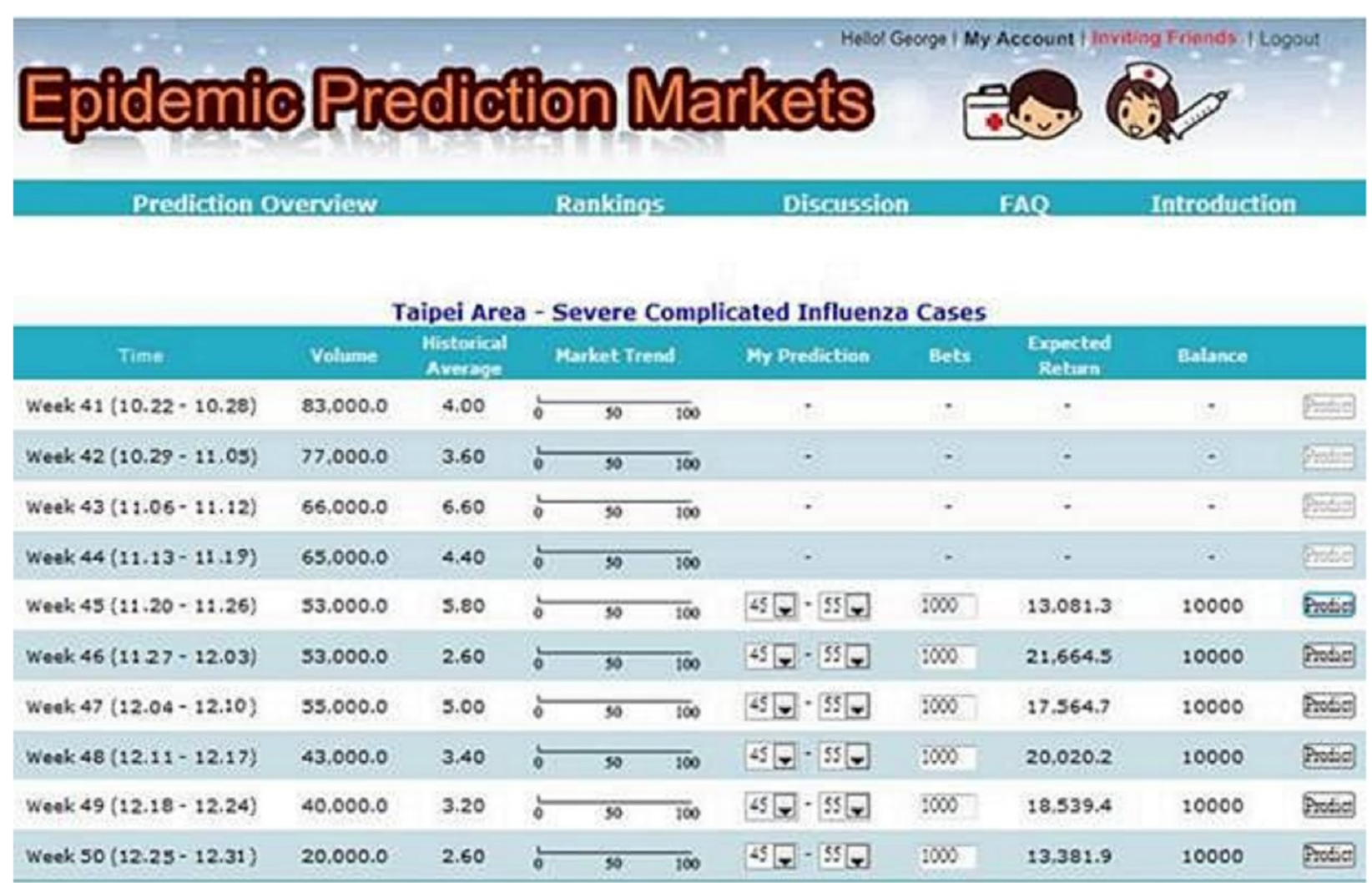

Fig. 1 Epidemic prediction markets

Google Flu Trends is not a prediction system. It is a surveillance system and does not provide surveillance signal for Taiwan. Nevertheless, Google Flu provides real-time surveillance information for the flu trend. Most prediction models provide neither real-time prediction nor surveillance information.

Recently, in a contest hosted by the Centers for Disease Control (CDC) of the USA to promote innovation in flu activity modeling and prediction, Shaman and his team

Table 1 The statistics of participants participating in prediction of five diseases indicators

\begin{tabular}{llll}
\hline Indicator & Participants & Predictions & Trading amount \\
\hline $\begin{array}{l}\text { Confirmed cases of dengue } \\
\text { fever }\end{array}$ & 84 & 4,395 & $29,503,332$ \\
$\begin{array}{l}\text { Confirmed cases of severe } \\
\text { complicated influenza case }\end{array}$ & 81 & 3,956 & $33,501,580$ \\
$\begin{array}{l}\text { Rate of enterovirus infection } \\
\text { Rate of influenza-like illness }\end{array}$ & 64 & 3,495 & $31,627,923$ \\
$\begin{array}{l}\text { Confirmed cases of severe } \\
\text { complicated enterovirus }\end{array}$ & 59 & 2,660 & $23,759,804$ \\
infection & & 4,291 & $23,199,675$ \\
\begin{tabular}{l} 
Total \\
\hline
\end{tabular} & 126 & 18,797 & $141,592,314$ \\
\hline
\end{tabular}

plugged digital data from CDC's influenza-like illness data as well as social media and internet search engine into a mathematical model and then calibrating the model to produce an accurate and reliable forecast for the timing, peak and intensity of the 2013-14 flu season. Shaman's team tested their model against actual flu activity that had already occurred during the season. By looking at the immediate past, Shaman and his team fined-tuned the model to better predict the future. However, the USA CDC has

Table 2 The statistics of participants participating in prediction of five diseases indicators in seven areas

\begin{tabular}{llll}
\hline Region & Participants & Predictions & Trading amount \\
\hline Taipei area & 64 & 3,511 & $24,875,745$ \\
Northern area & 50 & 2,596 & $21,166,549$ \\
Central area & 41 & 2,471 & $18,959,092$ \\
Southern area & 48 & 2,517 & $18,385,997$ \\
Kaohsiung- Pingtung area & 48 & 2,486 & $18,096,217$ \\
Eastern area & 32 & 2,570 & $18,748,079$ \\
Nationwide area & 49 & 2,646 & $21,360,636$ \\
Total & 126 & 18,797 & $141,592,314$ \\
\hline
\end{tabular}


Table 3 The statistics of participants participating in prediction of diseases indicators in eight weeks

\begin{tabular}{|c|c|c|c|c|c|}
\hline & Participants & Predictions & $\begin{array}{l}\text { Share of } \\
\text { predictions }\end{array}$ & $\begin{array}{l}\text { Trading } \\
\text { amount }\end{array}$ & $\begin{array}{l}\text { Share of } \\
\text { trading } \\
\text { amount }\end{array}$ \\
\hline $\begin{array}{l}\text { 0 week in } \\
\text { advance }\end{array}$ & 110 & 7,184 & $38 \%$ & $56,840,809$ & $40 \%$ \\
\hline $\begin{array}{l}1 \text { week in } \\
\text { advance }\end{array}$ & 89 & 2,289 & $12 \%$ & $15,620,775$ & $11 \%$ \\
\hline $\begin{array}{l}2 \text { weeks in } \\
\text { advance }\end{array}$ & 64 & 1,493 & $8 \%$ & $10,082,893$ & $7 \%$ \\
\hline $\begin{array}{l}3 \text { weeks in } \\
\text { advance }\end{array}$ & 51 & 1,169 & $6 \%$ & $8,179,810$ & $6 \%$ \\
\hline $\begin{array}{l}4 \text { weeks in } \\
\text { advance }\end{array}$ & 44 & 1,226 & $7 \%$ & $8,730,681$ & $6 \%$ \\
\hline $\begin{array}{l}5 \text { weeks in } \\
\text { advance }\end{array}$ & 38 & 1,233 & $7 \%$ & $8,807,299$ & $6 \%$ \\
\hline $\begin{array}{l}6 \text { weeks in } \\
\text { advance }\end{array}$ & 34 & 1,478 & $8 \%$ & $11,148,133$ & $8 \%$ \\
\hline $\begin{array}{l}7 \text { weeks in } \\
\text { advance }\end{array}$ & 32 & 2,725 & $14 \%$ & $22,181,914$ & $16 \%$ \\
\hline Total & 126 & 18,797 & $100 \%$ & $141,592,314$ & $100 \%$ \\
\hline
\end{tabular}

not yet officially used any model to predict the flu activity and will continue to explore the possibilities of flu forecasting.

We spent three months to present our methodology of epidemic prediction markets (EPM) to and collaborate with the Taiwan $\mathrm{CDC}$ on the project drafting. In the real world of Taiwan, we were told by the CDC of Taiwan during this Taiwan CDC-sponsored project drafting period that it was very difficult to predict the trend of three particular epidemic diseases in Taiwan: influenza, dengue fever and enterovirus. In the past, voluntary medical doctors reported their cases to the CDC as early as they could, but the results were not always complete and timely. Therefore, the CDC told us clearly that they relied on the historical average number of disease cases or rate (AVG) to portray the trend of epidemic diseases in Taiwan. That was why we used the AVG as the benchmark for the comparison of prediction accuracy for the EPM.

However, we will show that historical average data serve the purpose of surveillance rather than prediction after comparing their results with the prediction markets. Given the volatility of the infectious diseases, historical average is unlikely to be an effective prediction mechanism. Taiwan CDC understood that these forecasts were not accurate, but they did not have better methods to predict the trend of epidemic diseases in the real world. To solve this problem, efforts have been made to introduce other methods to forecast the spread of infectious diseases. This Taiwan CDC-sponsored project was one of them.

Prediction markets-initiated by the University of Iowa's Electronic Market in 1988 to predict a future event-have been proven useful for the continuous and real-time forecasting of infectious diseases [2, 3]. This paper tried to construct an epidemic prediction market (EPM) system to provide accurate, continuous and realtime prediction system for five indicators of three epidemic diseases for eight weeks ahead of the target week and seven regions around Taiwan. Since the AVG is currently the only method adopted by the CDC to depict the trend of epidemic diseases in Taiwan, we assessed the accuracy of the EPM prediction against that of the AVG.

The EPM we constructed not only provided accurate and real-time predictions as other prediction markets did for other diseases, but also improved the Iowa Influenza Market (IIM), so far the most successful prediction market of epidemics, in duration, space, the number of diseases, and the method of transaction. In addition, we

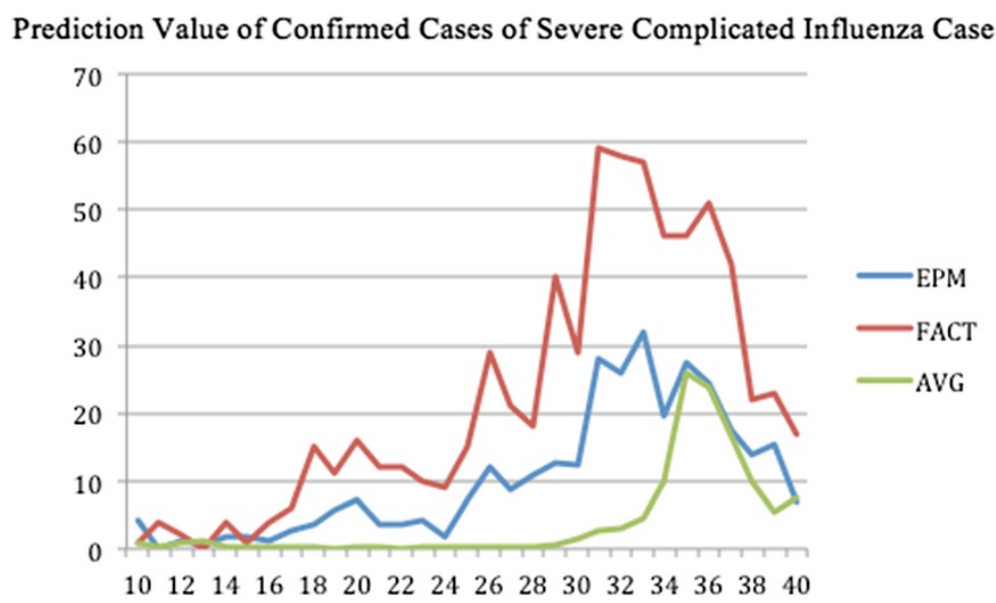

Fig. 2 Prediction value of confirmed cases of severe complicated influenza case 


\section{Prediction Value of Confirmed Cases of Dengue Fever}

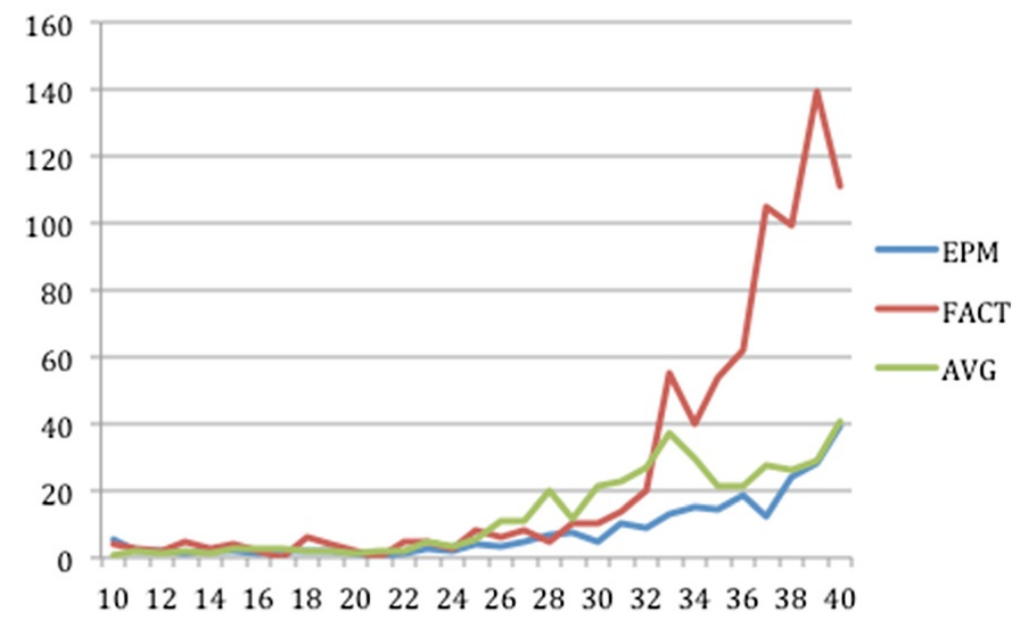

Fig. 3 Prediction value of confirmed cases of dengue fever

were told by the Taiwan CDC that it was very difficult for Taiwan CDC to predict the trend of these three particular epidemic diseases in Taiwan: influenza, dengue fever and enterovirus. It is thus a good case to test the capability of prediction markets. Located in the subtropical climate region, Taiwan is subject to easy outbreaks of epidemics of infectious diseases. Global warming and international transportation further increases the frequency and periodicity of these diseases, hence the difficulty to predict them $[7,8]$.

Infectious diseases can spread across any region, but Taiwan has not been a member of the World Health Organization. There is a surveillance system of infectious diseases in Taiwan. To build up a Taiwan's virus surveillance network, Taiwan $\mathrm{CDC}$ has so far commissioned 8 medical centers spread in all parts of Taiwan since 1999, and to have each set up a contract laboratory of diagnostic virology for detecting, organizing, and reporting suspected enterovirus cases as well as severe influenza ones. Besides, a separate sentinel physicianbased surveillance system was also established with doctors assigned almost in each and every district and township across Taiwan, and a domestic virus strain database was assembled. However, there is no effective prediction system for these epidemic diseases. Accordingly, filling this gap by accurate data regarding Taiwan's infectious diseases is of critical importance.

This paper will demonstrate the effectiveness of the EPM predictions of influenza, enterovirus, and dengue fever by comparing them with the historical averages released by the Taiwan government. The next two sections will describe our method and findings.

\section{Methods}

A prediction market, operating like a futures market, is a forecasting mechanism capable of processing the dynamic

Prediction Value of Confirmed Cases of Severe Complicated Entervirus Infection

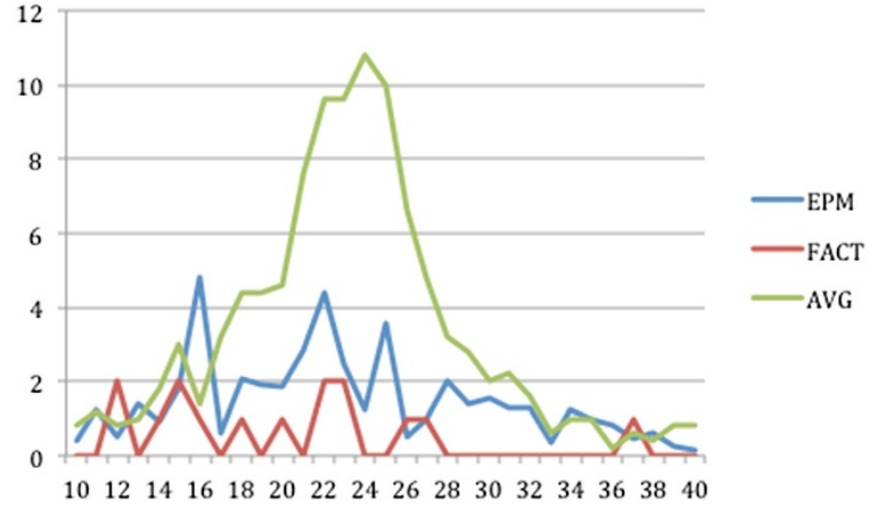

Fig. 4 Prediction value of confirmed cases of severe complicated entervirus infection 


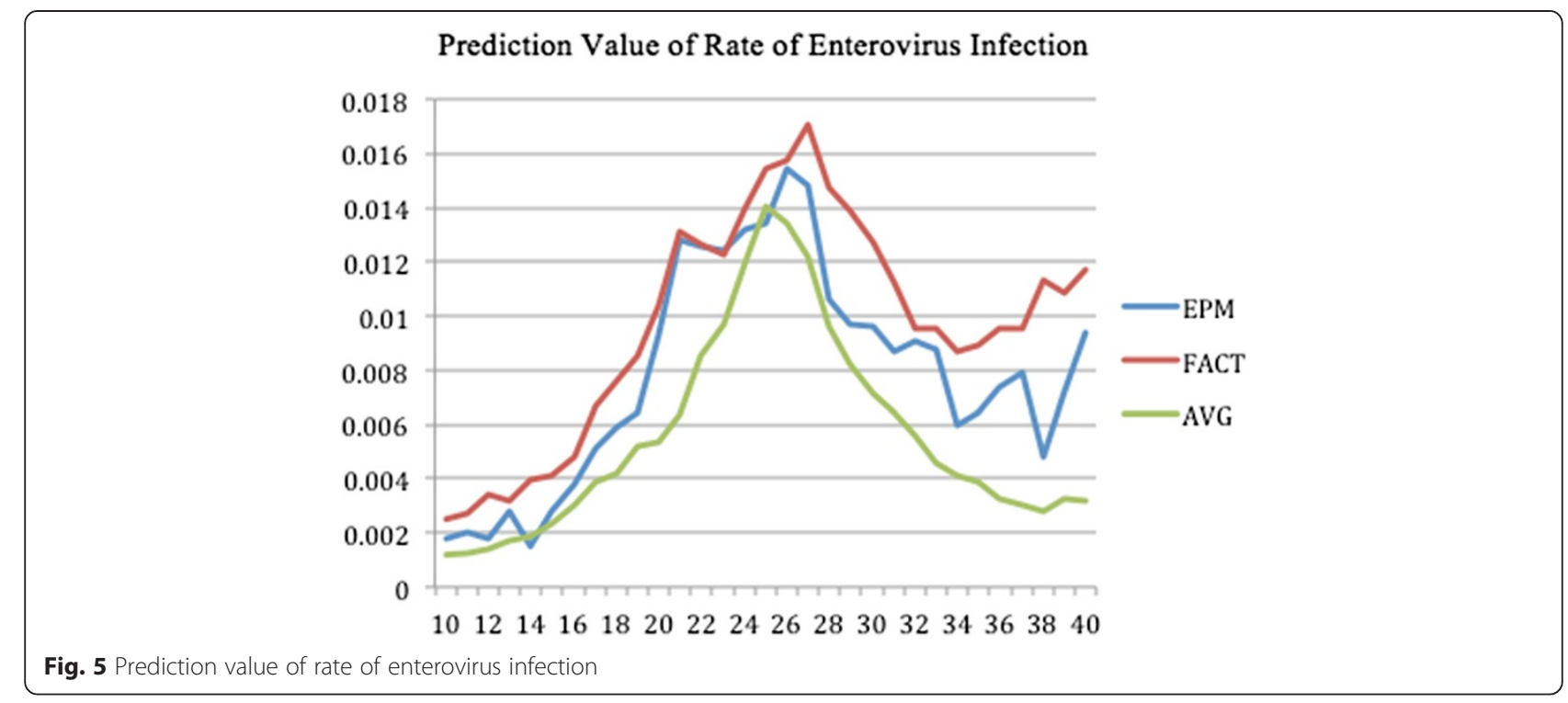

aggregation of dispersed information from various participants $[9,10]$. During the trading period, new information is continuously absorbed by the traders and reflected on the market prices. On the expiry date of the futures contracts, the settlement price will be determined by the outcome of the prediction event [11].

In the recent two decades, prediction markets have been proven empirically to be remarkably accurate in forecasting future events [12], such as elections [13-15], sports competitions $[16,17]$ and movie box offices [18-20]. Prediction markets have two major characteristics that are advantageous in prediction accuracy over traditional methods of prediction. The first is the incentive structure of reward and punishment, which induces participants to provide real and effective information and reduces market manipulation in most situations [21, 22]. The second is the continuous update of information for the predicted events, so that participants conduct trade online to provide real-time information.

We designed and built the first Epidemic Prediction Markets (EPM) system sponsored by the CDC of Taiwan (See Fig. 1). EPM invited medical professionals in Taiwan to participate. The registered members of EPM were encouraged to predict three epidemics with five indicators: (1) confirmed cases of severe complicated influenza case, (2) confirmed cases of dengue fever, (3) confirmed cases of severe complicated enterovirus infection, (4) rate of enterovirus infection, and (5) rate of influenza-like illness (ILI).

In order to improve trading liquidity and efficiency to aggregate information, EPM adopted market scoring rules (MSR) as the trading mechanism, instead of continuous double auction (CDA), which is often used by the stock or

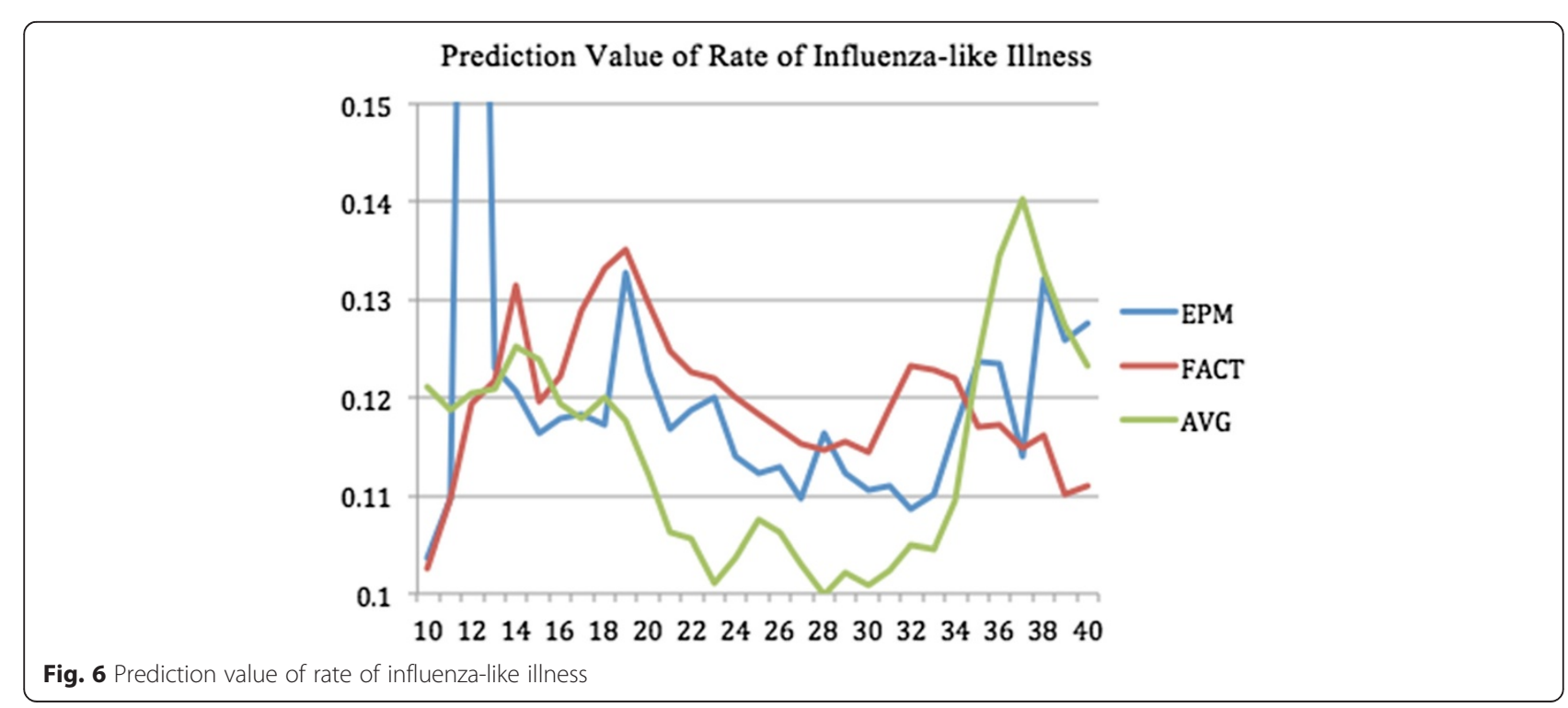




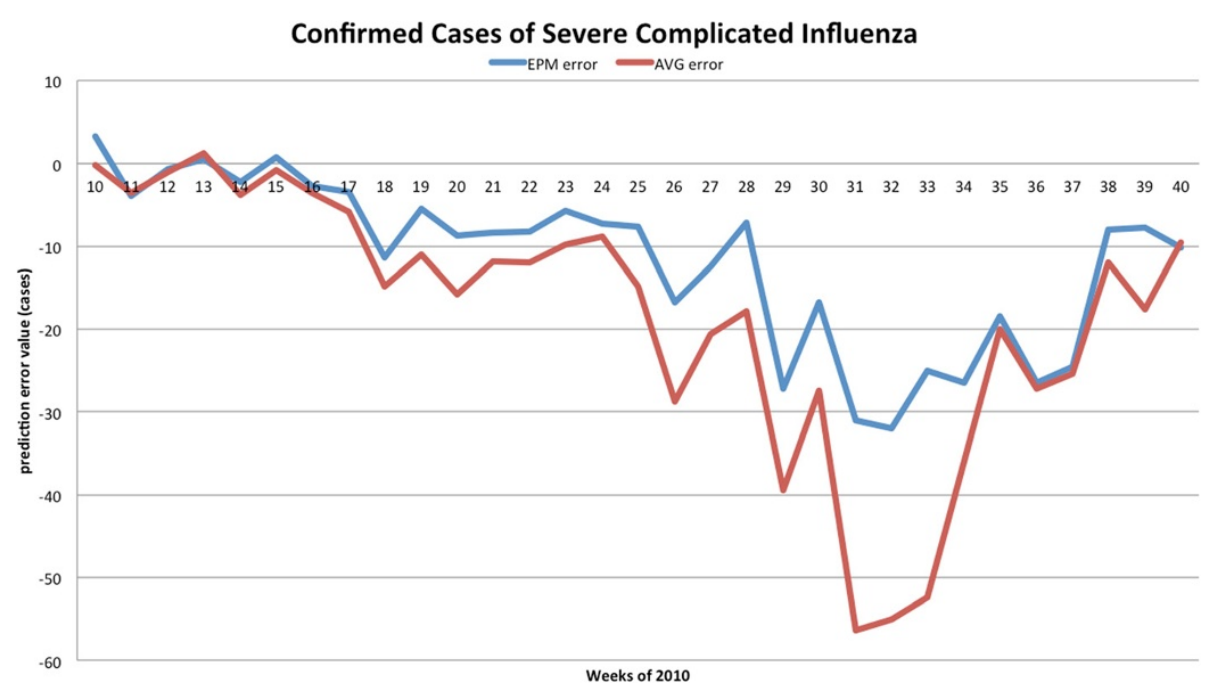

Fig. 7 Prediction error value for confirmed cases of severe complicated influenza

futures markets. Compared with CDA, MSR can avoid the thin market problems [23, 24]. This is very important to EPM as medical professionals are often too busy to actively participate in the trading.

According to the rules of EPM system, each participant was given 10,000 health dollar $(\mathrm{H} \$)$ for each prediction event, and they were encouraged to predict the three epidemics with five indicators for the target week and seven weeks in advance and in seven areas of Taiwan. Health dollar is trading instrument to provide valuable information of epidemic diseases in the epidemic prediction market. Participants with accurate prediction would gain positive credits, and vice versa. Each trader will be ranked by the net balance of positive credits for her/his performance in the predictions. For every month of trading period, active participants would be rewarded with three awards of USD 33. Outstanding participants would be rewarded with awards of USD 1,000, USD 666, and USD 333, along with prize certificates from the CDC of Taiwan.

In this paper, we compared the forecast accuracy of two forecasting methods: the first is the historical average number of disease cases or rate (AVG) for the same weeks from 2005 to 2009. The second is the predictive value of the EPM. Since the AVG is currently the only method adopted by the CDC to depict the trend of epidemic diseases in Taiwan, we compare our prediction results with that of the effective alterative method (AVG)

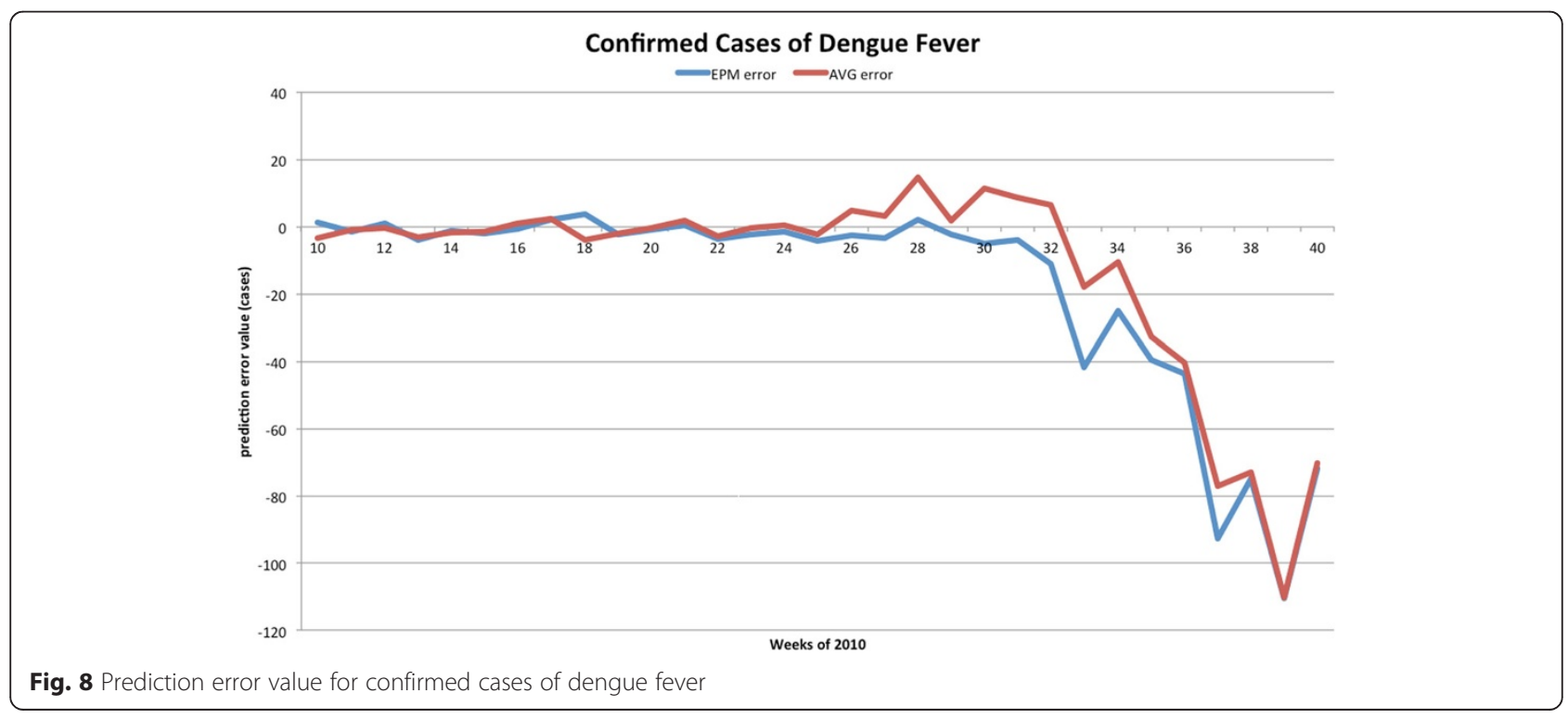




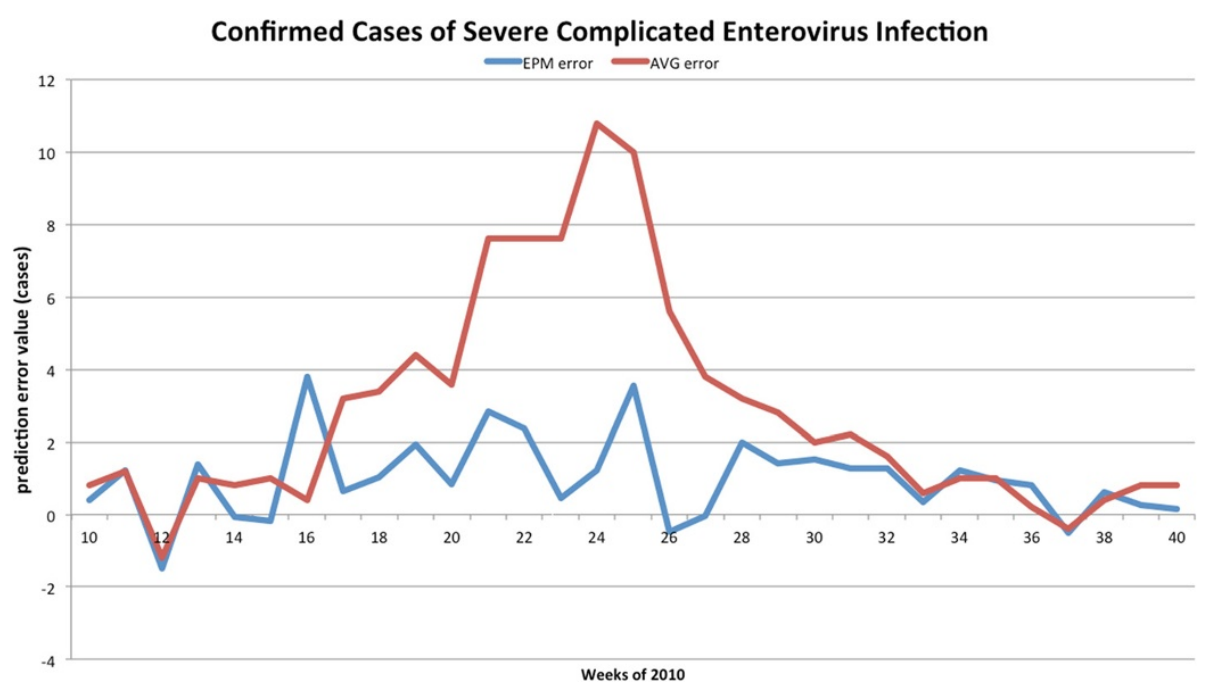

Fig. 9 Prediction error value for confirmed cases of severe complicated enterovirus infection

in the real world of Taiwan. In order to compare the accuracy, we calculate the prediction error values of these two methods as follows:

Prediction_error_value $=$ Predicted_value - Actual_value

Lower prediction error value means higher prediction accuracy, and vice versa. When the prediction error value of EPM is less than that of AVG, the number of wins for EPM is to increase by one. Therefore, the winning ratio is calculated as follows:

Winning_ratio $=$ Wins/Total_number_of_predictions

\section{Results}

\section{Participation of EPM}

From the 10th week (March 7-13) to the 40th week (October 3-9) of 2010, 630 medical professionals registered with this EPM. Nevertheless, only 126 members traded the prediction events of diseases indicators. The composition of these participants are: 48 nurse specialists, 23 doctors, 13 medical inspectors, four pharmacists, three nurses, two Chinese medicine practitioners and 33 other professionals. Regarding institutions, 40 participants were from public hospitals and another 40 from private hospitals; 21 from Taiwan's Ministry of Health and its related units; 7 from clinics; 4 from local public health bureaus; the rest 14 participants from other units. In terms of areas where the participants worked, 38 participants came from Taipei area; 29 from southern area; 21 from northern area; 15 from Kaohsiung-Pingtung area in the south of Taiwan; 12 from eastern area; and 11 from central area. Tables 1

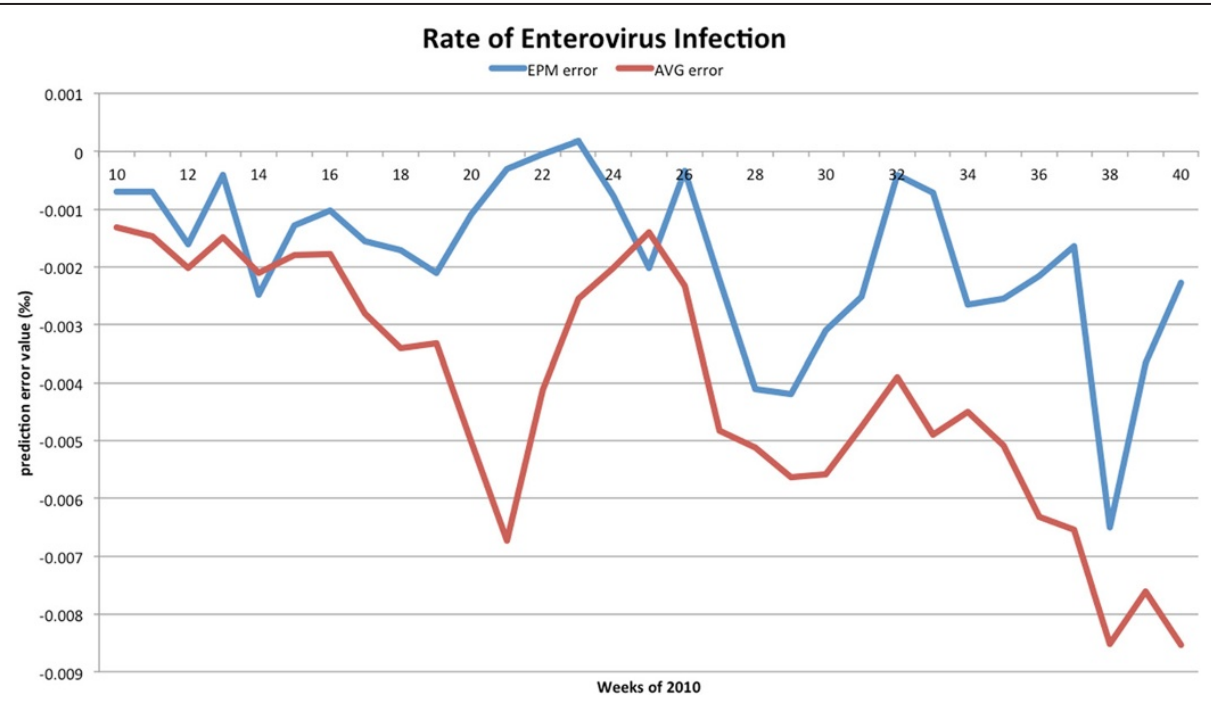

Fig. 10 Prediction error value for rate of enterovirus infection 


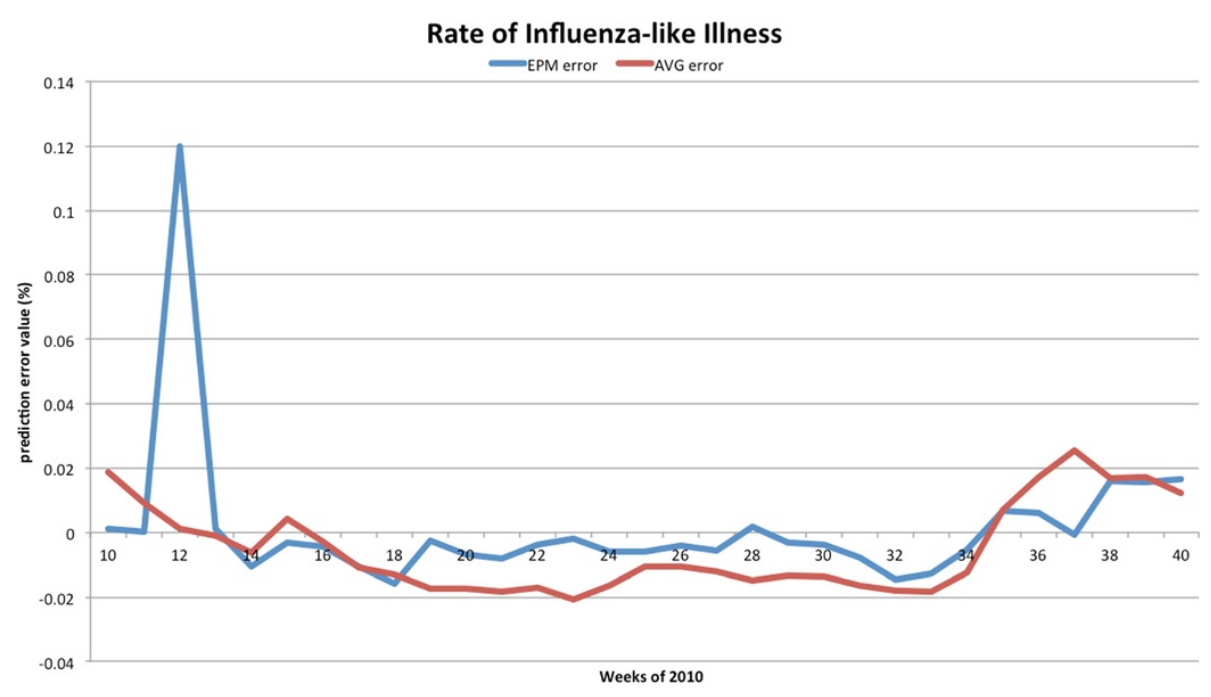

Fig. 11 Prediction error value for rate of influenza-like illness

and 2 show the statistics of participants participating in prediction of five diseases indicators and seven areas.

In terms of the statistics of participants participating in prediction of diseases indicators in different weeks in advance of the target week, there were 110 participants participated in the target week with 7,184 predictions (38\% of total predictions) and trading amount of $\mathrm{H} \$ 56,840,809$ (40\% of total trading amount). For one week in advance of the target week, 89 participated with 2,289 predictions (12\% of total predictions) and trading amount of $\mathrm{H} \$ 15,623,775$ (11\% of total trading amount). For 2 weeks in advance, 64 participated with 1,493 predictions (8\% of total predictions) and trading amount of $\mathrm{H} \$ 10,082,893$ (7 \% of total trading amount). For the rest, please See Table 3 .
Winning ratios of two methods for all diseases and all areas

The forecasted curves of incidence of the various diseases under this study are shown from Figs. 2, 3, 4, 5 and 6. In addition, the prediction error results of two methods for five indicators of three infectious diseases are presented from Figs. 7, 8, 9, 10 and 11. If every week's prediction on each diseases indicator was regarded as a prediction event, there were 7,945 prediction events in total. Concerning the prediction performance, for the target week (0 week in advance), EPM was more accurate in 701 out of 1,085 prediction events than AVG and the winning ratio of EPM versus AVG was $64.6 \%$. EPM's winning ratio was $55.5 \%$ for 1 week in advance, $54.4 \%$ for 2 weeks in advance, $53.0 \%$ for 3 weeks in advance, $52.8 \%$ for

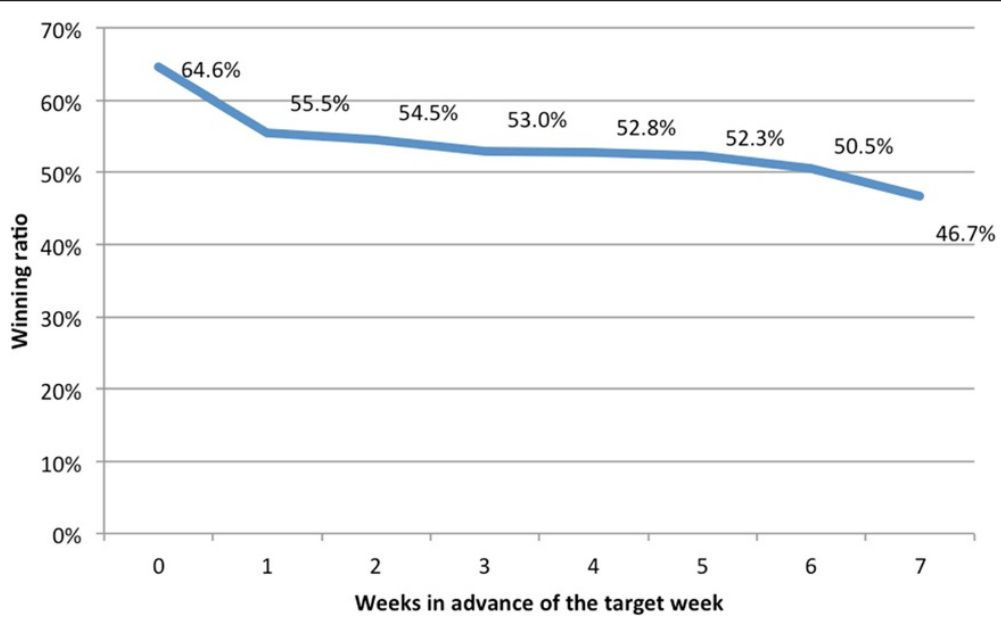

Fig. 12 The winning ratios of EPM versus AVG for all diseases indicators and all areas 
Table 4 Prediction error of EPM and AVG for five diseases indicators

\begin{tabular}{|c|c|c|}
\hline Indicator & EPM & AVG \\
\hline Confirmed cases of severe complicated influenza case & 11.955 & 18.206 \\
\hline Confirmed cases of dengue fever & 18.131 & 16.509 \\
\hline $\begin{array}{l}\text { Confirmed cases of severe complicated enterovirus } \\
\text { infection }\end{array}$ & 1.172 & 2.935 \\
\hline Rate of enterovirus infection & 0.00184 & 0.00411 \\
\hline Rate of influenza-like illness & 0.0104 & 0.0132 \\
\hline
\end{tabular}

4 weeks in advance, $52.3 \%$ for 5 weeks in advance and $50.5 \%$ for 6 weeks in advance. The winning ratio of EPM was only inferior to that of AVG for the 7 weeks in advance (See Fig. 12).

\section{Prediction errors and winning ratios of two methods for each disease and all areas}

For the prediction error of all five diseases indicators, EPM was more accurate than AVG except dengue fever confirmed cases. For the prediction of the confirmed cases of severe complicated influenza case, EPM's prediction error was 11.955 cases while AVG's prediction error was 18.206 cases. For the prediction of the confirmed cases of dengue fever, EPM's prediction error was 18.131 cases while AVG's prediction error was 16.509 cases. For the prediction of the confirmed cases of severe complicated enterovirus infection, EPM's prediction error was 1.172 cases while AVG's prediction error was 2.935 cases. For the prediction of the rate of enterovirus infection, EPM's prediction error was $0.00184 \%$ while AVG's prediction error was $0.00411 \%$. For the prediction of the rate of influenza-like illness, EPM's prediction error was $0.0104 \%$ while AVG's prediction error was $0.0132 \%$ (See Table 4).

\section{Cross analysis of prediction of five diseases and eight weeks}

For each disease indicator, there were 1,589 prediction events, including 217 prediction events for both target week and 1 week in advance of the target week, 210 for 2 weeks in advance, 203 for 3 weeks in advance, 196 for 4 weeks in advance, 189 for 5 weeks in advance, 182 for 6 weeks in advance, 175 for 7 weeks in advance. For the prediction for the confirmed cases of severe complicated influenza case, the rate of enterovirus infection, and the rate of influenza-like illness, the winning ratios of EPM versus AVG were obviously over $50 \%$ in all weeks. The winning ratios of EPM for these three indicators in the target week were 69.6, 83.9 and $76.0 \%$, respectively. Instead, for the prediction of the confirmed cases of dengue fever and the confirmed cases of severe complicated enterovirus infection, the winning ratios of EPM versus AVG were all below 50 \% (See Fig. 13).

\section{Cross analysis of prediction of five diseases and seven areas}

Table 5 shows the winning ratios of EPM versus AVG for the prediction of five diseases indicators in seven areas. First, except for the southern area and the Kaohsiung-Pingtung area (southern Taiwan), the winning ratios of EPM versus AVG for the prediction of the confirmed cases of dengue fever and severe complicated enterovirus infection were mostly less than $50 \%$. Second, in the southern area, the Kaohsiung-Pingtung area, and the eastern area, the winning ratios of EPM for the prediction of the rate of influenza-like illness were less than $50 \%$. Third, except confirmed cases of dengue fever, the highest winning ratios of EPM for predicting each indicators all occurred in the prediction of the nationwide area.

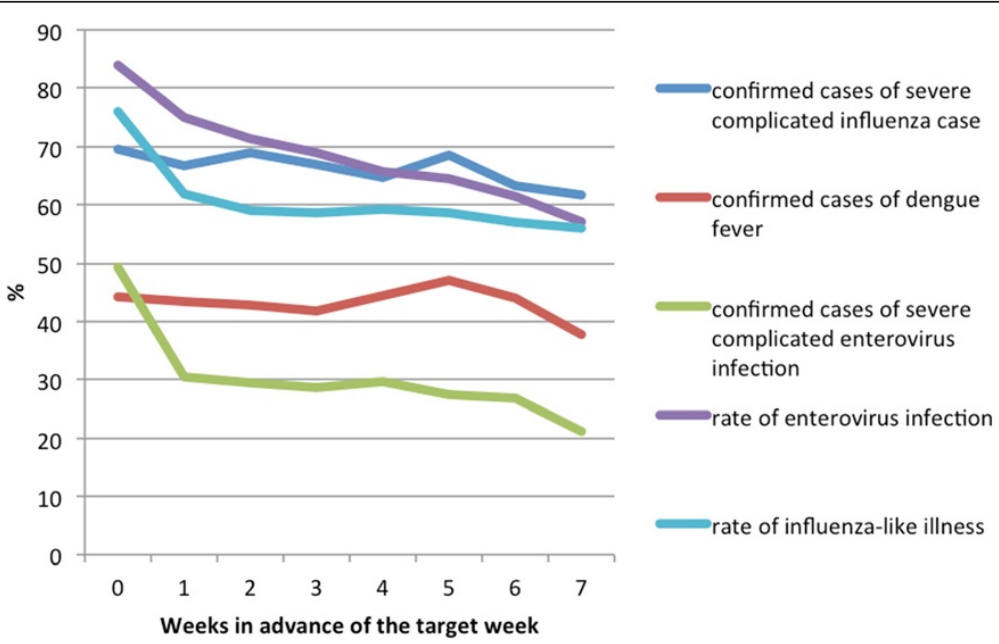

Fig. 13 The winning ratios of EPM versus AVG for each diseases indicators and all areas 
Table 5 The winning ratios of EPM versus AVG for five diseases indicators in seven areas

\begin{tabular}{|c|c|c|c|c|c|c|c|c|c|c|c|c|c|c|}
\hline & \multicolumn{2}{|c|}{ Taipei area } & \multicolumn{2}{|c|}{ Northern area } & \multicolumn{2}{|c|}{ Central area } & \multicolumn{2}{|c|}{ Southern area } & \multicolumn{2}{|c|}{ Kaohsiung-Pingtung area } & \multicolumn{2}{|c|}{ Eastern area } & \multicolumn{2}{|c|}{ Nationwide area } \\
\hline & $\begin{array}{l}\text { All } \\
\text { events }\end{array}$ & $\begin{array}{l}\text { arget } \\
\text { veek }\end{array}$ & $\begin{array}{l}\text { All } \\
\text { events }\end{array}$ & $\begin{array}{l}\text { Target } \\
\text { week }\end{array}$ & $\begin{array}{l}\text { All } \\
\text { events }\end{array}$ & & $\begin{array}{l}\text { All } \\
\text { events }\end{array}$ & & $\begin{array}{l}\text { All } \\
\text { events }\end{array}$ & & $\begin{array}{l}\text { All } \\
\text { events }\end{array}$ & & $\begin{array}{l}\text { All } \\
\text { events }\end{array}$ & $\begin{array}{l}\text { Target } \\
\text { week }\end{array}$ \\
\hline 11 & $71.4 \%$ & $.7 \%$ & $\%$ & $71.0 \%$ & $\%$ & $54.8 \%$ & $\%$ & $\%$ & 6 & $\%$ & $\%$ & $67.7 \%$ & $\%$ & $90.3 \%$ \\
\hline or 2 & $40.5 \%$ & $355 \%$ & $.7 \%$ & $645 \%$ & 880 & $45.2 \%$ & $4.2 \%$ & 548 & 4 & $548 \%$ & $\%$ & $22.6 \%$ & $06 \%$ & 230 \\
\hline ndicator 3 & $24.2 \%$ & $48.4 \%$ & $25.1 \%$ & $35.5 \%$ & $40.1 \%$ & $54.8 \%$ & $36.1 \%$ & $54.8 \%$ & $22.0 \%$ & $64.5 \%$ & $9.3 \%$ & $12.9 \%$ & $58.6 \%$ & $74.2 \%$ \\
\hline dicator 4 & $89.4 \%$ & 0250 & $72.7 \%$ & 0680 & $71.4 \%$ & $90.3 \%$ & $68.3 \%$ & 7100 & 5200 & 7120 & $50.7 \%$ & $67.7 \%$ & 7810 & $93.5 \%$ \\
\hline ndicator 5 & $67.4 \%$ & $80.6 \%$ & $71.8 \%$ & $80.6 \%$ & $68.7 \%$ & $74.2 \%$ & $40.1 \%$ & $58.1 \%$ & $44.9 \%$ & $71.0 \%$ & $61.7 \%$ & $87.1 \%$ & $73.1 \%$ & $80.6 \%$ \\
\hline verage & $58.6 \%$ & $65.2 \%$ & $59.3 \%$ & $69.7 \%$ & $55.2 \%$ & $63.9 \%$ & $51.8 \%$ & $61.3 \%$ & $44.8 \%$ & $66.5 \%$ & $42.2 \%$ & $51.6 \%$ & $66.3 \%$ & 74.2 \\
\hline
\end{tabular}

Note: Indicator 1: confirmed cases of severe complicated influenza case Indicator 2: confirmed cases of dengue fever

Indicator 3: confirmed cases of severe complicated enterovirus infection

Indicator 4: rate of enterovirus infection

Indicator 5: rate of influenza-like illness

\section{Cross analysis of prediction of seven areas and eight weeks}

Table 6 shows the winning ratios of EPM versus AVG for the prediction of all diseases indicators for eight weeks in seven areas. First, except the prediction for the target week, the winning ratios of EPM for the Kaohsiung-Pingtung and Eastern areas were less than $50 \%$. Second, after 4 weeks in advance of the target week in the southern area, the winning ratios of EPM were barely above $50 \%$. Third, the highest winning ratios of EPM for the prediction of each week all occurred in the nationwide area.

\section{Discussion}

One should note that the winning ratios of EPM for the diseases have some variance in the target week. They are: (1) $69.6 \%$ for the confirmed cases of severe complicated influenza case; (2) $44.2 \%$ for the confirmed cases of dengue fever; (3) $49.3 \%$ for the confirmed cases of severe complicated enterovirus infection; (4) $83.9 \%$ for the rate of enterovirus infection; (5) $76.0 \%$ for the rate of influenza-like illness. Among these diseases, (1), (2), and (3) are lower because they are "lagging indicators"-the data must undergo laboratorial tests to be confirmed for around one month. As this takes time, such information is hard to collect and confirmed in advance (according to Taiwan CDC).

Our EPM also improved the prediction market developed by IIM in several ways. While IIM made its prediction for one disease taking place in one State for 14 weeks, our EPM covered five diseases in seven areas in Taiwan for 31 weeks; IIM's forecasting target was the surveillance signals, ours was the number of cases and rates. Our cases were more complicated, which increased the difficulty of prediction, yet we managed to achieve a high winning ratio when compared with the historical average.

Furthermore, the success of EPM confirms the marginal trader theory: unlike the general traders who passively take the market price, marginal traders are those who submit their orders when the market price is about to be made; they may be few in number but exert a strong impact on the eventual price $[25,26]$. In EPM, 126 participants predicted 7,945 events, proving EPM to be a successful mechanism of information collection. Particularly, a few medical professionals with significant

Table 6 The winning ratio of EPM versus AVG for the prediction of all diseases indicators for eight weeks in seven areas

\begin{tabular}{|c|c|c|c|c|c|c|c|}
\hline & Taipei area & Northern area & Central area & Southern area & Kaohsiung-Pingtung area & Eastern area & Nationwide area \\
\hline Target week & $65.2 \%$ & $69.7 \%$ & $63.9 \%$ & $61.3 \%$ & $66.5 \%$ & $51.6 \%$ & $74.2 \%$ \\
\hline 1 week in advance & $58.7 \%$ & $60.0 \%$ & $61.3 \%$ & $54.8 \%$ & $42.6 \%$ & $43.2 \%$ & $67.7 \%$ \\
\hline 2 weeks in advance & $57.3 \%$ & $58.7 \%$ & $56.7 \%$ & $52.0 \%$ & $44.0 \%$ & $43.3 \%$ & $68.7 \%$ \\
\hline 3 weeks in advance & $58.6 \%$ & $59.3 \%$ & $53.1 \%$ & $50.3 \%$ & $40.7 \%$ & $42.1 \%$ & $66.9 \%$ \\
\hline 4 weeks in advance & $59.3 \%$ & $58.6 \%$ & $52.1 \%$ & $50.7 \%$ & $40.7 \%$ & $41.4 \%$ & $66.4 \%$ \\
\hline 5 weeks in advance & $62.2 \%$ & $58.5 \%$ & $53.3 \%$ & $48.1 \%$ & $40.7 \%$ & $37.8 \%$ & $65.2 \%$ \\
\hline 6 weeks in advance & $56.2 \%$ & $55.4 \%$ & $51.5 \%$ & $49.2 \%$ & $38.5 \%$ & $40.0 \%$ & $63.1 \%$ \\
\hline 7 weeks in advance & $49.6 \%$ & $52.0 \%$ & $46.4 \%$ & $45.6 \%$ & $42.4 \%$ & $36.0 \%$ & $55.2 \%$ \\
\hline Average & $58.6 \%$ & $59.3 \%$ & $55.2 \%$ & $51.8 \%$ & $44.8 \%$ & $42.2 \%$ & $66.3 \%$ \\
\hline
\end{tabular}


confidence of disease information were sufficient to lead the price. In fact, three top traders comprised $86.3 \%$ of total won credits after predicting 7,945 events.

These accomplishments aside, some issues of epidemic prediction markets are worth studying in the future. First, some may question the validity of our findings by raising the possibility of self-defeating predictions-if government and people are convinced of the prediction results, would they not take preventive measures so that the original prediction becomes inaccurate? Selfdefeating prediction, even if possible, is least likely to discredit prediction markets because they are more likely than any other forecasting tools to produce realtime and continuous revisions of its predictions. That is, efforts of preventive measures should be reflected in the market price.

The second issue is also about the social responses to EPM. To make their predictions more reliable, prediction markets should have as many participants as possible even if some of them are not professionals. Yet we cannot exclude the possibility that results of an open EPM can cause a dreadful response from the public if the targets are infectious diseases. How to improve public welfare without precipitating social panic is an important topic for the proponents of prediction markets to consider.

\section{Conclusions}

EPM outperformed current methods in two ways. First, while EPM offered predictions, web search or sentinel physicians system provided only surveillance. Second, EPM predicted infectious diseases more accurately than historical averages. When compared with the historical average of the previous five years, the winning ratio of EPM on influenza, Dengue fever and enterovirus of the target weeks of 2010 is $64.6 \%$. There is also evidence indicating that the effectiveness of EPM improved as the forecast time drew nearer to the deadline.

In addition, the variance of EPM predictions across regions is insignificant. Across diseases, the winning ratios of the national forecasting are significantly higher than the regional ones. This is an important finding because it shows that even if participants are making imperfect predictions, they can be aggregated into more accurate ones by the mechanism of prediction markets.

\section{Competing interests}

The authors declare that they have no competing interests.

\section{Authors' contributions}

All authors conceived of and participated in the design of the study, carried out the case study and literature review. TCC performed the statistical analysis. CYT drafted the manuscript. JWL revised the manuscript. All authors read and approved the final manuscript.

\section{Acknowledgements}

This research was sponsored by both the Centers for Disease Control and the Ministry of Science and Technology, R.O.C. (Taiwan). The publication fee was financed by the Institute of Political Science at Academia Sinica, Taiwan.

\section{Author details}

${ }^{1}$ Graduate Institute of Development Studies, National Chengchi University, 64, Zhi-Nan Road, Sec. 2, Wenshan, Taipei 11605, Taiwan. ${ }^{2}$ Center for Prediction Markets, National Chengchi University, Taipei, Taiwan. ${ }^{3}$ Institute of Political Science, Academia Sinica, Department of Political Science, National Chengchi University, Taipei, Taiwan.

Received: 30 August 2014 Accepted: 5 August 2015

Published online: 11 August 2015

\section{References}

1. Chretien J-P, George D, Shaman J, Chitale RA, McKenzie FE. Influenza forecasting in human populations: a scoping review. PLOS ONE. 2014;9(4), e94130. doi:10.1371/journal.pone.0094130.

2. Polgreen PM, Nelson FD, Neumann GR. Using prediction markets to forecast trends in infectious diseases. Microbe. 2006;1:459-65.

3. Polgreen PM, Nelson FD, Neumann GR. Use of prediction markets to forecast infectious disease activity. Clin Infect Dis. 2007:44:272-9.

4. Nsoesie EO, Beckman RJ, Shashaani S, Nagaraj KS, Marathe MV. A simulation optimization approach to epidemic forecasting. PLoS ONE. 2013;8(6), e67164. doi:10.1371/journal.pone.0067164.

5. Ginsberg J, Mohebbi MH, Patel RS, Brammer L, Smolinski MS, Brilliant L. Detecting influenza epidemics using search engine query data. Nature. 2009:457:1012-5.

6. Polgreen PM, Chen Y, David M, Pennock DM, Nelson FD. Using internet searches for influenza surveillance. Clin Infect Dis. 2008;47:1443-8.

7. World Health Orgnization. Climate change and infectious disease. http://www.who.int/globalchange/climate/summary/en/index5.html, April 25, 2015.

8. Tatem AJ, Rogers DJ, Hay SI. Global transport networks and infectious disease spread. Adv Parasitol. 2006;62:293-343.

9. Forsythe R, Nelson F, Neumann GR, Wrigh J. Anatomy of an experimental political stock market. Am Econ Rev. 1992:82:1142-61.

10. Snowberg E, Wolfers J, Zitzewitz E. Partisan impacts on the economy: evidence from prediction markets and close elections. Q J Econ. 2007;122(2):807-29.

11. Mansk CF. Interpreting the predictions of prediction markets. Econ Lett. 2006;91(3):425-9.

12. Berg JE, Nelson D, Rietz TA. Prediction market accuracy in the long run. Int J Forecast. 2008:24(2):285-300.

13. Bruggelambert G. Information and efficiency in political stock markets: using computerized markets to predict election results. Appl Econ. 2004;36:753-68.

14. King R. Workers, place your bets. Bussiness Week. 2006, August 2. http://www. bloomberg.com/bw/stories/2006-08-02/workers-place-your-bets, May 7, 2015.

15. Walker D. Predicting presidential election results. Appl Econ. 2006;38:483-90.

16. Luckner S, Schröder J, Slamka C. On the forecast accuracy of sports prediction markets. In: Gimpel H, Jennings NR, Kersten GE, Ockenfels A, Weinhardt C, editors. Negotiation, auctions, and market engineering. Berlin, Heidelberg: Springer-Verlag Berlin Heidelberg; 2008. p. 227-34.

17. Spann M, Skier B. Sports forecasting: a comparison of the forecast accuracy of prediction markets, betting odds and tipsters. J Forecast. 2009;28:55-72.

18. Foutz NZ, Jank W. The wisdom of crowds: pre-release forecasting via functional shape analysis of the online virtual stock market. SSRN: http://ssrn.com/abstract=1432444, July 4, 2007.

19. Gruca TS. The IEM movie box office market integrating marketing and finance using electronic markets. J Mark Educ. 2000;22:5-14.

20. Gruca T, Berg JE, Cipriano M. The effect of electronic markets on forecasts of new product success. Inf Syst Front. 2003;5(1):95-105.

21. Camerer CF. Can asset markets be manipulated? A field experiment with racetrack betting. J Polit Econ. 1998;106(3):457-82.

22. Rhode PW, Strumpf KS. Manipulating political stock markets: a field experiment and a century of observational data, available at: http://www.unc.edu/ cigar/papers/ManipNBER.pdf, May 1, 2008.

23. Hanson R. Combinatorial information market design. Inf Syst Front. 2003;5(1):105-19. 
24. Hanson R. Logarithmic market scoring rules for modular combinatorial information aggregation. J Prediction Markets. 2007;1:3-15.

25. Forsythe R, Rietz TA, Ross TW. Wishes, expectations and actions: a survey on price formation in election stock markets. J Econ Behav Organ. 1999;39:83-110.

26. Oliven K, Rietz TA. Suckers are born but markets are made: individual

rationality, arbitrage, and market efficiency on an electronic futures market. Manag Sci. 2004;50:336-51.

Submit your next manuscript to BioMed Central and take full advantage of:

- Convenient online submission

- Thorough peer review

- No space constraints or color figure charges

- Immediate publication on acceptance

- Inclusion in PubMed, CAS, Scopus and Google Scholar

- Research which is freely available for redistribution 